\title{
Das Migrationsthema bei Gianmaria Testa: zur ethischen und ästhetischen Hinterlassenschaft des Liedermachers aus dem Piemont
}

\author{
Nora MOLL (Rom)
}

\section{Summary}

This article focuses on the work of the Italian Cantautore Gianmaria Testa, who died prematurely this year. In particular, it analyses his concept album Da questa parte del mare (2006), which deals with the issue of migration. In the eleven songs of this album, Testa establishes a link between the migrations of the past and the present, in order to show that human feelings and behaviour do not change in 'times of crises'. As the article tries to point out, Testa's musical style and lyrics are the result of an intense aesthetic work. It is based on ethical principles and empathy, it creates intertextual relations with contemporary literary texts and with the Italian tradition of songwriters and reaches a remarkable expressiveness.

Levigare le parole / fino alla trasparenza fino al limite sottile / di fragilità e di rischio per sentirle finalmente suonare / al tocco delle dita o tagliarvisi le labbra / o raccoglierne i cocci muti e riprovare (Testa 2016,89)

Am 30. März 2016 verstarb Gianmaria Testa. Der über die Grenzen seiner Heimat hinaus bekannte Cantautore erlag mit 58 Jahren einem Hirntumor. Zuletzt hatte sich der Künstler nach seinem Rückzug von der Bühne im Mai 2015 über seine Erkrankung öffentlich geäußert, äußerst gefasst und voller Hoffnung. ${ }^{1}$ Die musikalische Laufbahn des Liedermachers, der aus einer einfachen Familie von Landwirten im italienischen Piemont stammte, begann relativ spät: 1993 und 1994 wurde er beim Festival musicale di Recanati mit dem ersten Preis ausgezeichnet, 1995 erschien unter dem Titel Mongolfières bei der französischen Plattenfirma Label Bleu sein erstes Album, dem bis 2013 weitere sechs Original- und zwei Life-Alben folgten. ${ }^{2}$ Noch bevor Gianmaria Testa in Italien von der Kritik gewürdigt wurde, hatte er es bereits Mitte der 1990er Jahre zuerst in Frankreich zum Erfolg gebracht. Zur Popularität des piemontesischen Liedermachers beim französischen Publikum, dessen musikalischer Stil in 
mancher Hinsicht mit dem von Georges Brassens verwandt ist, hatte u.a. seine Freundschaft mit dem Marseiller Schriftsteller Jean-Claude Izzo beigetragen. Dieser schätzte die intimen Sonoritäten des italienischen Chansonniers so sehr, dass er in seinem 1997 erstmals veröffentlichten Roman Marins perdus sogar ein Lied von Gianmaria Testa zitierte. Neben dem für seine mediterranen Noir-Romane berühmten Schriftsteller Izzo, der mit zahlreichen musikalischen Textzitaten Testas die nostalgische Atmosphäre seiner Romane bereichert, pflegte der italienische Liedermacher auch zu Erri De Luca eine fruchtbare Freundschaft. Ein Beispiel für die künstlerische Zusammenarbeit mit dem gebürtigen Neapolitaner, der zu den politisch engagiertesten Autoren im heutigen Italien zählt, ist das Projekt Don Chisciotte e gli invincibili von 2007, das unter Mitwirkung des Klarinettisten Gabriele Mirabassi auf mehreren italienischen Bühnen aufgeführt wurde.

Dieser kurze Blick auf das künstlerische Wirken von Gianmaria Testa lässt bereits erahnen, wie sehr sich darin - über die Jahre hinweg in zunehmendem Maße - musikalische und literarische Erfahrungen überkreuzen und gegenseitig potenzieren. Es geht dabei nicht nur um rein intertextuelle Bezüge, sondern um kreative Zusammenarbeit, um den Austausch von Ideen und Weltanschauungen, die den piemontesischen Cantautore sowohl in ästhetischer als auch in ethischer Hinsicht beeinflusst haben. Im Folgenden soll nun zum einen aufgezeigt werden, wie Gianmaria Testas Zuwendung zum Migrationsthema von der aktiven Rezeption von Erri De Lucas Gedichtsammlung Solo andata (2005) profitiert, zum anderen aber auch, wie der piemontesische Liedermacher bei dem infrage stehenden Album Da questa parte del mare (2006) auch an eine schon zuvor erarbeitete Poetik anknüpft. Aus textkritischer Sicht erweist sich in diesem Album die Kunst der Canzone bei Testa als überaus vielfältig. Im Vergleich zu anderen italienischen Liedermachern ${ }^{4}$ ist die von ihm aufgezeigte Verbindung zwischen den Auswanderungsbewegungen der italienischen Bevölkerung und den seit Ende der 1980er Jahre fast exponentiell zunehmenden Einwanderungswellen nach Italien schließlich als höchst innovativ einzuschätzen.

Tatsächlich kann man beobachten, dass Testa zwischen der Herausgabe des musikalisch sehr geglückten Albums Altre latitudini (2003) und der darauffolgenden CD Da questa parte del mare (2006) einen interessanten thematischen Sprung vollzieht, ohne aber auf die Wiederaufnahme früherer Themen ganz zu verzichten. Vor der Herausgabe des eben genannten Concept-Albums hatte sich der piemontesische Künstler vorwiegend dem Thema der Liebe gewidmet, wofür Kompositionen wie "Gli amanti di Roma“" (aus dem Album Il valzer di un giorno, 2000), „Preferisco cosi“ und "Come di pioggia“ (aus dem Album Altre latitudini, 2003) als Beispiele genannt werden können. In anspruchsvollen musikalischen Arrangements wird mit verschiedensten Lied- und Tanzrhythmen experimentiert, die stets dem unausgesprochenen Gebot der Schlichtheit und der Eleganz folgen. Testas samtig tiefe Stimme stützt sich ganz nach Cantautore-Tradition auf die eigene Gitarrenbegleitung. Der Hörer wird in Situationen des Alltags geführt, in denen die Dimensionen des Unerwarteten, aber auch des Unscheinbaren erforscht werden: „Tutto è già qui, / anche se non si vede. / Tutto è già qui, / nascosto tra le pieghe. / E se ci stupirà / sarà soltanto com è / come certe novità / che sapevamo giä“, lautet beispielsweise die erste Strophe von „Il valzer di un giorno“, 
dem Titelsong des gleichnamigen Albums von 2000, in dem die Behauptung, alles sei doch schon dagewesen und nichts könne ein wahres Staunen hervorrufen, schließlich doch nur als Auftakt zur Einführung des Liebesthemas dient. Die beglückte Leichtigkeit des Stücks wird auch musikalisch durch einen rhythmischen Wechsel in den Dreivierteltakt untermalt. Neben den Epiphanien des Glücks, die bei Testa häufig mit Liebesgefühlen verbunden sind, erscheinen in den Kompositionen vor 2006 auch Momente der Resignation, des Liebesverlustes und der Einsamkeit; Bilder einer schlichten, aber niemals ,grauen' Gegenwart aus der Perspektive der „soligna stanza“ des lyrischen Ichs. Der häufig vollzogene Vergleich mit dem aus derselben italienischen Region stammenden Liedermacher Paolo Conte ist jedoch sowohl aus thematischer als auch aus stilistischer Sicht kritisch zu sehen, und beruht wohl doch lediglich auf dem ähnlich tiefen Timbre von dessen Stimme. Tatsächlich sind Contes Liedertexte eher von einem Erzählstil geprägt, der den Hörer in die vergangene Zeit der späten 1940er und der 1950er Jahre, d.h. in die Jugendzeit des italienischen Cantautore, entführt und von dem „diritto alla memoria di un tempo andato" (Tirone / Giovanetti 1996, 139) massiv gebraucht macht. Dem „io impudico“ (Tirone / Giovanetti 1996, 140) Contes, das sich durch eine provokative, z.T. gewollt banale und stark extrovertierte Sprache äußert, steht Testas lyrisch orientiertes ,io solitario' gegenüber, das die Intimität und die schlichte Intensität der Gefühle an sprachliche Eleganz bindet. Selten verlassen Testas Lieder dabei das Register der klassischen Hochsprache. Nur in Ausnahmefällen dringen sie in den Bereich bestimmter Jargons oder der in der Geschichte der italienischen Canzone oft verwendeten Dialekte vor. ${ }^{5}$ Auch der thematische Kurswechsel, den der piemontesische Liedermacher, wie schon erwähnt, mit seinem 2006 veröffentlichten Album Da questa parte del mare ${ }^{6}$ vollzieht, lässt die sprachlich-stilistische Ebene mehr oder weniger unberührt. Testa macht dort von denselben Stilmitteln wie in den vorangehenden Alben Gebrauch, die nun aber zum ersten Mal auf ein stark ethisch orientiertes Thema angewandt werden: die Migration von Menschen über die Mittelmeerroute oder über den Atlantik gestern wie heute.

Die Texte der elf Lieder dieses Albums sind erneut Momentaufnahmen, aber diesmal auf offener Straße, aus der Sicht von Menschen, die in einem Land "auf dieser Seite des Meers", also an der nördlichen Seite des Mittelmeers leben, das schon seit Beginn der 1990er Jahre besonders von Albanern und in den darauffolgenden Jahren zunehmend von Flüchtlingen aus den Krisengebieten Afrikas und des Nahen Ostens durchquert wurde. Wie Testa in einem Interview von 2006 mit Isabella Maria Zoppi berichtet, entwickelte sich die Idee des Albums aus einem Erlebnis während eines Badeurlaubs. Die Geretteten und die Toten aus der alltäglichen Presseberichterstattung seien ihm plötzlich „in Fleisch und Blut" erschienen und hätten ihn das Leiden der , illegal ${ }^{6}$ eingereisten Migranten - der ,clandestini' - mit einem veränderten Blick erfassen lassen:

Sono partiti in due da un qualche porto del Nord Africa, clandestini nascosti nella stiva di un cargo. A due terzi del viaggio li hanno scoperti e buttati a mare. Li ha raccolti un peschereccio nell'Alto Adriatico. Nessun tipo di soccorso a bordo. Li hanno scaricati come zavorra dentro un gommone attraccato a duecento metri da una spiaggia di 
Puglia. Quando li hanno portati a riva, per uno di loro non c'è stato più niente da fare. L'altro, dopo, ha raccontato. Erano i primi anni '90. Non ho scritto per loro. Non ne sarei capace. Ho scritto per me e per quelli che, come me, stanno da questa parte del mare. (Zoppi 2007, 116)

Wie aus den Worten Testas deutlich hervorgeht, war die Entscheidung, sich mit dem Migrationsthema zu beschäftigen, von Anfang an von dem als Notwendigkeit erachteten Ansinnen bestimmt, bei den italienischen Landsleuten hinsichtlich der immer stärker politisierten Frage der ,illegalen Einwanderung' (,immigrazione clandestina') ein verändertes, durch Empathie und menschliche Anteilnahme geprägtes Bewusstsein herzustellen. Dabei lässt sich Testa nicht so sehr von statistischen Daten und journalistisch aufgearbeiteten Berichten inspirieren, sondern von persönlichen Begegnungen mit Einwanderern wie auch von der ,Begegnung' mit Erri De Lucas Buch Solo andata (2005). Die Originalität von Testas Album liegt jedenfalls nicht im Migrationsthema selbst, weist doch die Emigrationsthematik in der italienischen Canzone-Tradition eine massive Präsenz auf und ist auch die Thematisierung der Immigration der letzten Jahrzehnte, wie vorhin erwähnt, längst kein Einzelfall mehr. Als originell und innovativ kann aber die Art und Weise gelten, wie der piemontesische Liedermacher den literarischen Prätext in seine Lieder einbindet, wie er das Narrativ der Migration über das gesamte Album hinweg ausdehnt und dabei kontinuierlich einen Zusammenhang zwischen Emigration von gestern und Immigration von heute herstellt.

Ein ähnlicher Rückgriff auf einen literarischen Text zur Migration war fast zehn Jahre zuvor von Fabrizio De André vollzogen worden, als sich dieser von einem der ersten bekannteren Texte der italienischen Migrationsliteratur ${ }^{7}$ inspirieren ließ, um sein berühmtes Lied „Prinçesa“ (in Anime salve, 1996) zu verfassen. Es handelt sich dabei um eine musikalisch-textuelle Neuverarbeitung des gleichnamigen autobiographischen Romans des brasilianischen Transsexuellen Farias de Albuquerque, den dieser zusammen mit dem ehemaligen Rotbrigadisten und Dokumentarfilmregisseur Maurizio Jannelli im Gefängnis verfasst hatte (de Albuquerque / Jannelli 1994). ${ }^{8}$ Obwohl die Autorenschaft dieses ,Urtextes der zu Beginn der 1990er Jahre in Italien aufkommenden Migrationsliteratur nicht ganz unumstritten ist, hatte die ,intertextuelle Reise“ (Fracassa 2012, 114) des Romans Princesa die italienische Öffentlichkeit vermehrt auf das persönliche Drama bestimmter Einwanderergruppen gelenkt, ein Drama, das sowohl in de Albuquerques Autobiographie als auch in De Andrés Lied aus der Ich-Perspektive dargestellt wird. Allerdings ging es in diesem Fall gleichzeitig um das besonders bei der musikalischen réecriture von Princesa stark im Vordergrund stehende Schicksal eines Vertreters der Transgender-Problematik, die mit der von De André so oft beschriebenen und teilweise idealisierten Problematik der Prostitution verknüpft wurde. In anderen Worten, der mit der Migration einhergehende Identitätsverlust und das als Konsequenz der Fremde beschriebene Abdriften von Farias de Albuquerque in die Sphäre der Kriminalität und der Drogensucht werden in De Andrés Fassung dieser Autobiographie im Vergleich zum Original zugunsten des Themas Transsexualität und Prostitution ausgeblendet. 
Im Gegensatz zu dem eben beschriebenen Fall geht es bei dem hier zu analysierenden Beispiel um einen Prätext, in dem in dem ein bekannter (wenn auch nicht zum kulturellen Establishment zählender) italienischer Autor das Thema Migration verarbeitet, wobei der intertextuelle Bezug hier viel loser ist. Bei Erri De Lucas Gedichtsammlung Solo andata konzentriert sich Testa besonders auf deren gleichnamigen ersten Teil, der abwechselnd Solound Gruppenrezitation in Anlehnung an die Aufführungspraxis eines griechischen Chors beinhaltet. ${ }^{9}$ In diesem durch seine narrative Anlage deutlich episch gehaltenen Text verwendet Erri De Luca weitgehend die erste Person Plural und wählt für die Darstellung der Geschehnisse vor und während einer Überfahrt auf dem Mittelmeer archaisierende Bilder, um der verletzten Würde der Flüchtlinge bildlich Ausdruck zu verleihen. Hier ein Beispiel aus der mit „coro“ betitelten Episode:

Gli uomini hanno lasciato le preghiere a terra, del viaggio non ha colpa il Dio di ognuno.

Nessuna invocazione, supplica di aiuto, da qui solo un saluto al re dell'universo.

Se eravamo a terra in queste notti cantavamo

Per le mandrie portate in altopiano.

Tenevamo lontani i leoni con il canto, le donne curavano il fuoco nel cerchio di pietra.

Qui non si posa in terra l'ombra dei nostri corpi, siamo polvere alzata, un odore di aceto in una fiasca vuota.

Siamo deserto che cammina, popolo di sabbia, ferro nel sangue, calce negli occhi, un fodero di cuoio.

Molte vite distrutte hanno spianato il viaggio, passi levati ad altri spingono i nostri avanti. (De Luca 2005, 24)

In Gianmaria Testas Lied „Seminatori di grano“, einem Largo für Vokal, Gitarre, Celli und Klarinette, ist eine Kontinuität mit dieser archaisierenden Darstellung des Herkunftslands der Migranten zu beobachten, die durch eine lexikalische Rekurrenz belegt wird: der Ausdruck „altipiano/i“ (also ,Hochebene/n', in beiden Texten zwecks Hervorhebung im Kursiv) als Bezeichnung für den imaginären Lebensraum ,davor', der auch in semantischer Hinsicht im drastischen Gegensatz zum Schicksal der Einwanderer nach dem Verlassen ihrer Heimat steht. 


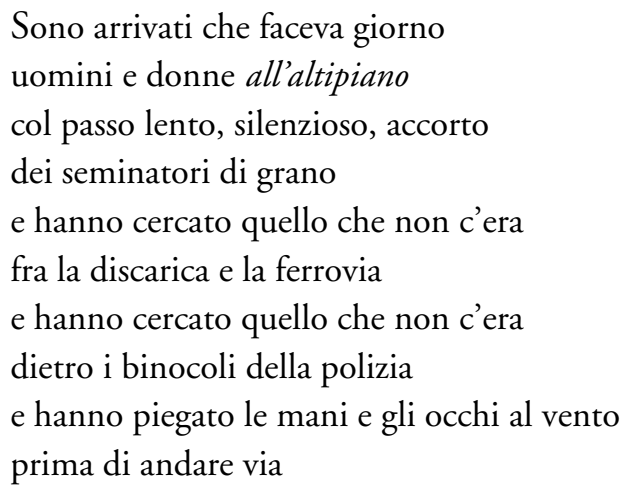

Fino alla strada e con la notte intorno sono arrivati dall'altipiano uomini e donne con lo sguardo assorto dei seminatori di grano e hanno lasciato quello che non c'era alla discarica e alla ferrovia e hanno lasciato quello che non c'era agli occhi liquidi della polizia e hanno disteso le mani contro il vento che li portava via (Testa 2016, 7) ${ }^{10}$

Im Gegensatz zu Erri De Lucas Gedicht ist dieser Text in der dritten Person gehalten und arbeitet weniger mit epischen Modulen als mit fast fotografischen Momentaufnahmen. Er macht den Verlust einer traditions- und naturgebundenen Lebensweise durch weitere Kontraste deutlich: Die als „seminatori di grano“, also als „Getreidesäer“ bezeichneten Auswanderer suchen etwas nicht Vorhandenes („quello che non c'era“) zwischen „der Müllhalde und der Eisenbahn“, also an Orten, die eine desolate Modernität symbolisieren, und werden von Polizisten mit „wässrigen Augen“ beobachtet. ${ }^{11}$ Die in Testas Liedtext als langsam, leise und gefasst beschriebenen Schritte der Auswanderer verweisen außerdem auf die kontrollierten Bewegungen der Landwirte, die noch während der Kindheit des Liedermachers aus dem Piemont das Getreide per Hand aussäten, Bewegungen, in denen „c’era qualcosa di definitivo e fiero, e c'era anche un'intrinseca speranza di raccolto propizio“ (Testa 2016, 3-4). So formuliert es Testa selbst in dem kurz nach seinem Tod im April 2016 erschienenen Buch zum Album Da questa parte del mare, das eine Reihe von autobiographisch geprägten Einführungen zu den jeweiligen Liedtexten enthält. Man erfährt dort unter anderem, dass Testas Vater dieselbe stolze Hoffnung der Landwirte, zu denen auch er selbst gehörte, in einem berühmten Bild von Pellizza da Volpedo, Il quarto stato (von 1901), wiedergefunden habe. ${ }^{12}$ Die eigenen Kindheitserinnerungen und die figurative Darstellung eines archaischen, noch stark rural geprägten Italiens werden also von Testa, im Einklang mit De Lucas Gedicht, auf die Einwanderer von heute übertragen. Diesen wird 
eine Würde und eine innere Kraft zugeschrieben, die in der heutigen Konsumgesellschaft nur noch schwer zu finden sei.

Das zweite, in f-Moll gehaltene Stück des Albums stützt sich nach einem orientalisch stilisierten Klarinettenvorspiel auf eine vorwiegend der Rockmusik entlehnten Begleitung mit E-Gitarre. Hier stehen thematisch die Wut und die Enttäuschung des Migranten im Vordergrund, der dazu gezwungen ist, sich als ,clandestino', als illegal Einreisender, den verbrecherischen Schleppern des Mittelmeers anzuvertrauen. „Come un ladro di notte / in mano a un ladro di mare", heißt es bezeichnender Weise in der dritten Strophe des Lieds, das den Titel „Rrock“ trägt und einem Violinisten aus Albanien gewidmet ist, der Jahre nach seiner Ankunft in Italien in einem Verkehrsunfall umgekommen war. Die dumpfe Verzweiflung und die Angst vor der nächtlichen Überfahrt werden durch eindrucksvolle Bildfragmente und durch einen von der indirekten Rede durchkreuzten inneren Monolog ausgedrückt:

Ma non era così

che mi avevano detto il mare

no non era così

e poi tanto di notte

cosa vuoi mai vedere

qui c'è uno che grida

che dice ch"è tardi e bisogna partire

qui c'è uno che grida ch'è tardi

e si deve partire.

E mio padre non c'è

è rimasto da solo a masticare la strada

perché dice che tanto sarà guerra comunque

e dovunque si vada.

L'ho lasciato alla porta di casa

che sputava per terra

come fosse un saluto

l'ho lasciato che sputava per terra

come se fosse un saluto.

Ma non era così

che mi credevo di andare

no non era così

come un ladro, di notte

in mano a un ladro di mare

e mio padre alla porta di casa che guardava per terra

come se avesse saputo

e mio padre che guardava per terra

come se avesse saputo. (Testa 2016, 13-14) 
Das Abschiednehmen des lyrischen Ichs erscheint in dieser Komposition noch umso dramatischer, als der Vater wortlos in der Haustür steht und als einzigen Gruß resigniert auf den Boden spuckt. In der von dem Text vermittelten Hoffnungslosigkeit schwingt darüber hinaus ein ganzer Generationenkonflikt mit, der ,jenseits des Meers“ durch den Mythos des wohlhabenden Okzidents verschärft wird, aber schon im Moment der Entscheidung auszubrechen oder ,durchzubrennen' (wofür im arabischen das Word harraga verwendet wird ${ }^{13}$ ) mit einer ersten Niederlage verbunden ist.

Nach „Forse qualcuno domani“, einem Lied in G-Dur, das in musikalischer Hinsicht eine lyrische Pause darstellt, in der über den Identitätsverlust vieler „namenloser“ Migranten meditiert wird, folgt eines der trauervollsten Stücke des Albums mit dem Titel „Una barca scura". Es handelt sich hierbei um einen zwischen Dur- und Molltonarten schwankenden Trauergesang, der wie im Dialog mit den ausufernden Verzierungen der Klarinettenmelodie einen „canto lento e sussurrato gemello al coro muto degli annegati“ (Zoppi 2009, 42) herstellt; ein Gesang, der Tausenden von Ertrunkenen gewidmet ist, aber sich nicht anmaßt, irgendjemanden trösten zu können:

In fondo al mare canta una sirena

tutta la notte canta e canta piano

per chi la vuol sentir si sente appena

in fondo al mare canta una sirena.

E in mezzo al mare va una barca scura

che ha perso il vento, perso alla sua vela

e chi la sta a aspettar l'aspetta ancora

in mezzo al mare va una barca scura.

E in fondo al mare, in fondo al mar profondo

ci lascio il canto mio che non consola

per chi è partito e si è perduto al mondo

in fondo al mare, in fondo al mar profondo. (Testa 2016, 29)

Im Gegensatz zu diesem Liedtext, in dem fast obsessiv die assonantischen Ausdrücke „in fondo al mar" und „mar profondo" wiederholt werden und dessen symmetrische Reimstruktur (drei Vierzeiler mit Kreuzreimen, die an erster und vierter Stelle jeweils identische Reime enthalten) den auf Inhaltsebene evozierten statischen Effekt noch verstärkt, ist das Lied mit dem Titel „Al mercato di Porta Palazzo“ (an achter Stelle im genannten Album) eines der freudigsten und energischsten. Es entstand, als Testa erneut Vater geworden war, und ist ein musikalisches Experiment, das sich bewusst von Balladen von Georges Brassens oder Fabrizio De André inspirieren lässt (Testa 2016, 49-52). Die im Dreivierteltakt gehaltene Canzone, deren Rhythmus auch an den italienischen saltarello erinnert, erzählt bezeich- 
nenderweise von der Geburt eines Kindes, und zwar unter freiem Himmel und mitten auf dem Turiner Markt von Porta Palazzo. Das Lied beginnt mit einem Blick auf ein Szenarium, das allerdings weniger an das multikulturelle Turin von heute als an vergangene Zeiten erinnert, als die Frauen noch lange schwarze Röcke und die Männer Stöcke trugen:

\author{
Al mercato di Porta Palazzo \\ fanno la fila, fanno la fila \\ le femmine da ragazzo \\ fanno la fila, fanno l'andazzo \\ e si lasciano indovinare \\ sotto le gonne, sotto le gonne \\ e si lasciano indovinare \\ sotto le gonne, le gonne nere. (Testa 2016, 53)
}

Nach einer weiteren Ortsbeschreibung in der zweiten Strophe („E sopra il molo del caricamento") - einer scherzhaften Hommage an Fabrizio De André, da sich Piazza del Caricamento und die Mole in Genova und nicht in Turin befinden - wird in der dritten Strophe der Ballade das zentrale Ereignis eingeleitet: „dalla coda del caricamento / qualcuno grida, qualcuno grida / e nella piazza di Porta Palazzo / fra le ragazze si rompe la fila“. Tatsächlich ist mitten in dem Gewimmel von Menschen und Röcken, an einem eisigen Wintertag, ein Kind zur Welt gekommen:

\author{
Che ce n'è una sdraiata per terra \\ sopra la neve che svapora \\ ce n'è una sdraiata per terra \\ e tutte le altre le fanno corona \\ e alle sette e quarantacinque \\ era già nato, era già fuori \\ alle sette e quarantacinque \\ l'hanno posato sul banco dei fiori. (Testa 2016, 54)
}

Selbstverständlich wird in diesem Text nicht von einer ,normalen' Geburt berichtet, sondern es geht hier darum, in ironischen Tönen und mit Entlehnungen aus dem Volksjargon („una“ für „una donna“; „era già fuori“ neben „era già nato“, etc.) eine illegale Geburt ohne Dokumente und ohne "qualcosa da dichiarare“ (ein Ausdruck aus der altertümlich klingenden Zollsprache) zu beschreiben. Doch wie so oft, wenn ein Kind geboren wird, so ist auch diesem Ereignis eines „sgravidamento / sul suolo pubblico comunale“ eine erstaunliche Schönheit abzugewinnen, wenn in der vorletzten Strophe des Lieds das Neugeborene mit den auf dem Marktplatz feilgehaltenen Blumen metaphorisch gleichgestellt wird: 
Ma documenti non ce ne sono

E neanche qualcuno che dica niente

Solo la gente che tira e che stringe

Intorno ai garofani e alle gardenie

Documenti non ce n'è sono

E quasi più niente da documentare

Solo che un giorno di luna d'inverno

Tutta la piazza ha voluto il suo fiore. (Testa 2016, 54-55)

Während die bisher besprochenen Liedertexte den Versuch Testas verdeutlichen, das Immigrationsszenarium im Italien von heute mit Bildern und sprachlichen Anspielungen auf das Italien von gestern zu veranschaulichen, thematisiert das folgende Lied aus dem Album Da questa parte del mare die unmittelbar nach der nationalen Einheit Italiens von 1861 massiv einsetzende Auswanderung nach Übersee. Das mit „Ritals“ betitelte Lied in C-Dur wird von einem Cellochor eingeleitet und verwendet mehrere musikalische Motive, die auf den klassischen italienischen Canzonestil verweisen. Der Text setzt mit der adversativen Konjunktion „eppure“ ein, die man mit ,jedoch' bzw. ,und dennoch“ übersetzen kann und die somit auf eine implizit vorausgehende Überlegung hinweist, die etwa so klingen könnte: Niemand denkt daran, dass wir auch einmal Migranten waren, und dennoch...

Eppure lo sapevamo anche noi

l'odore delle stive

l'amaro del partire

lo sapevamo anche noi

e una lingua da disimparare

e un'altra da imparare in fretta

prima della bicicletta

lo sapevamo anche noi

e la nebbia di fiato alle vetrine

e il tiepido del pane

e l'onta di un rifiuto

lo sapevamo anche noi

questo guardare muto. (Testa 2016, 65)

Das durchwegs in zarten Durtonarten gehaltene Lied, das eine gewisse romantische Effusion nicht scheut, ruft die Erinnerung an die Zeit der Armut und der Schwierigkeit, sich an die Kultur der neuen Heimat zu gewöhnen, wach. Diese wird aus der Perspektive eines „noi“ vollzogen, das Kinderaugen hat, was elliptische Wendungen wie „prima della bicicletta" deutlich machen. Der Schmerz, den Gianmaria Testa hier zum Ausdruck bringt, ist ein verhaltener Schmerz: Er äußert sich in poetischen Bildern wie dem Atemhauch, der sich 
auf Schaufensterscheiben sammelt, hinter welchen sich unerreichbare Waren befinden, und dem „mageren Wohnen“ in der zweiten Strophe („e un abitare magro, magro“), das den Einwanderern nicht erlaubt, sich richtig zuhause zu fühlen („che non diventa casa“) (Testa, 2016, 65-66). Enigmatisch erscheint auf den ersten Blick der Titel des Liedes, „Rital“, der aber in der dem Text vorangestellten Anekdote eine Erklärung findet und an die anfangs erwähnte Freundschaft zu dem aus Marseille stammenden Schriftsteller Jean-Paul Izzo anschließt: ,Rital' ist im französischen Argot eine abwertende Bezeichnung für die italienischen Einwanderer nach Frankreich und spielt durch die Aussprache mit einem gerollten ,r' auf deren Schwierigkeit an, das gutturale französische , $\mathrm{r}^{\mathrm{k}} \mathrm{zu}$ erlernen. Izzo, der als Sohn eines aus der Provinz von Salerno stammenden Einwanderers und einer spanischen Mutter in Marseille aufgewachsen war, mag dieses Wort wohl oft gehört haben, obwohl er schon „vor dem Fahrradfahren“ perfekt Französisch sprechen konnte. ${ }^{14}$

In dieser dem Lied „Rital“ vorangestellten Erzählung von einem Treffen mit Jean-Paul Izzo berichtet Testa auch davon, wie er dem französischen Freund ein Lied von Roberto Murolo („Munasterio 'e Santa Chiara“) vorspielte, das in ihm unerwartete Erinnerungen weckte, weil sein Vater es sehr oft gesungen habe. Der biographische Verweis kann in diesem Kontext auch als Anlass dienen, die immens wichtige Rolle zu betonen, die die italienische Canzone des späten 19. und des 20. Jahrhunderts für die Identitätsvermittlung und -bewahrung unter Emigranten besaß ${ }^{15}$ - eine Rolle, die von der italienischen Literatur vor 1990 nur in beschränktem Maße erfüllt wurde. Nicht nur im Kontext der Volksliedtradition oder der neapolitanischen Canzone, sondern auch durch Liedermacher der 1960er und 1970er Jahre (u.a. Lucio Dalla, Francesco De Gregori, Luigi Tenco) und durch Cantautori, die in jüngsten Jahren vom Dialekt oder von alternativen Musikformen Gebrauch machen (Davide Van De Sfroos, der Rapper Kento), wurde an diesem Thema weitergearbeitet und entstanden neue ästhetische Dimensionen. Innerhalb dieses Panoramas nimmt Gianmaria Testa tatsächlich eine Sonderstellung ein: Die Verknüpfung des Themas der italienischen Emigration mit dem Thema der Immigration bzw. der Flucht nach Italien, die im öffentlichen Diskurs mittlerweile zwar angeschnitten wird, aber kaum zu politischen Konsequenzen geführt hat, spricht für ein klares ethisches und im weiten Sinne politisches Engagement Testas. ${ }^{16}$ Denn auch in Da questa parte del mare kommt es mit dem schon genannten „Ritals“ sowie den zwei letzten Liedern des Albums nur scheinbar zu einem nostalgischen Eintauchen in das italienische kulturelle Gedächtnis und die Liedtradition des Landes: „Miniera“, eine Canzone von 1929 über den Tod eines Minenarbeiters, ${ }^{17}$ erscheint in der expressiven und gleichzeitig verhaltenen Version Testas als Identifikationsangebot für jeden Einwanderer und lässt sich vor dem Hintergrund inakzeptabler Arbeitsbedingungen vieler illegaler Immigrantinnen und Immigranten auf die Situation im heutigen Italien ummünzen.

Um ein politisches Statement, dies sei nochmals betont, handelt es sich bei Testas Liedtexten aber nur in einem weiten Sinn. Ganz anders als in den jüngsten literarischen oder journalistischen Recherchen zu der „Zeit, als wir die Albaner waren“ (cf. Stella 2002) fehlt dem musikalisch-dichterischen Narrativ Testas jede Schärfe. Und nicht nur dies: Auch das bei Testas früheren Alben so stark vertretene Liebesthema wird mit dem Migrationsnarrativ 
verwoben, und zwar im sechsten und siebten Stück von Da questa parte del mare, ein aus musikalischer Sicht wohl etwas unscheinbares Liederpaar, das aber in thematischer Hinsicht eine interessante Nuance einführt, die wiederum auf die italienische Literaturtradition verweist. Im ersten dieser beiden Lieder, „Il passo e l'incanto“, wird im meditativen Monolog von einer Rückkehr nach Lampedusa aus der Sicht eines ehemaligen Bootflüchtlings erzählt, der sich an eine Frauenfigur erinnert, deren Wärme spendende Blicke dazu beigetragen hatten, ihn am Leben zu erhalten; im zweiten, mit „3/4“ betitelten Lied wird dieselbe Szene aus der Sicht eben dieser Frau wiederaufgerollt. Der hier deutlich im Vordergrund stehende Topos des ,rettungsspendenden Blicks', der trotz des Bezugs auf eine wahre Begebenheit eine klare Anspielung auf den italienischen Dolce stil novo darstellt, ist in diesem Fall allerdings nicht als rein intertextueller und kultivierter Verweis zu bewerten. Auch die künstlerische Darstellung des Flüchtlingsdramas wird bei Testa mit derselben Schlichtheit und Intensität vollzogen, die den sprachlichen wie musikalischen Stil des piemontesischen Liedermachers durchwegs charakterisieren, fußt aber auf einer sehr deutlichen Empathie mit den Betroffenen, die durch die weitgehende Verwendung der Ich-Perspektive verstärkt wird.

In diesem wie in den zuvor genannten Fällen dienen die intertextuellen Bezüge zur Gegenwartsliteratur und die Wiederaufnahme von Themen, die verschiedenen Literatur- und Liedtraditionen eigen sind, also vor allem der Empathiebildung, die vor dem Hintergrund der sich zunehmend verschärfenden Notlage im Mittelmeer eine besondere ethische Dimension erreicht. So ist, wie gezeigt wurde, Testas Bezugnahme auf De Luca zwar nur in einer Canzone, „Seminatori di grano“, als konkrete lexikalische Intertextualität fassbar, sie bestimmt jedoch als kontinuierlicher impliziter Verweis auf einen Ideenaustausch und einen ethischen Konsens zwischen den beiden Künstlern, auf das Weiterschreiben gemeinsamer Gedanken, das gesamte hier untersuchte Album. Dieses lässt Testa mit dem für ihn charakteristischen Grundton eines ethisch-humanitären, nicht aber im engeren Sinn politischen Engagements auch ausklingen. Die abschließende Canzone „La nostra città ist eine zarte Liebeserklärung an Testas ,kleine‘, fast unscheinbare Heimat Cavallermaggiore („Ѐ / la nostra città / una città piccola / e non ci passano i tram“; Testa 2016, 77), deren naturgeprägte Schönheit schlaglichtartig mit der von Trambahnen durchzogenen Großstadt Turin verglichen wird: eine Heimat, die sich in Gianmaria Testas Musik und in den bezugsreichen und einfühlvollen Texten auch denen zu öffnen scheint, die ihr eigenes Zuhause verloren haben.

\section{Endnoten}

1 Siehe das von der italienischen Tageszeitung La Repubblica veröffentlichte Interview von Michele Serra mit Gianmaria Testa vom 11. Mai 2015 (Serra 2015).

2 Für die vollständige Diskografie Testas verweise ich auf die offizielle Webseite des verstorbenen Künstlers: www.gianmariatesta.com/discografia/ (Zugriff 20.06.2016).

3 Der Roman des im Jahr 2000 frühzeitig verstorbenen französischen Schriftstellers wurde 2008 von Simone Gandolfo und Fabio Beccaccini in ein Theaterstück umgearbeitet, das unter dem 
Titel Rien a signaler (una finestra sul Mediterraneo) im Festival Teatrale Borgo Verezzi debütierte und zu dem Testa die Bühnenmusik komponiert hatte.

4 Weitere repräsentative italienische Liedermacher, die sich mit der Migrationsthematik auch hinsichtlich der neueren Immigration kreativ auseinandergesetzt haben, sind Fabrizio De André, Roberto Vecchioni, Ivano Fossati, Samuele Bersani und Fiorella Mannoia.

5 Neben der Verwendung des piemontesischen Dialekts in „La Ca Sla Conlin-A“ (aus dem Album Extra Muros, 1996) sei die Neuinterpretation eines neapolitanischen Klassikers wie „Na Stella“ (Altre Latitudini, 2003) genannt.

6 Produzioni Fuorivia / Radio Fandango / Harmonia Mundi; Produktionsleitung: Paola Farinetti; Musiker: Gianmaria Testa (Vokalist; Gitarre), Enzo Pietropaoli (Kontrabass), Gabriele Mirabassi (Klarinette), Claudio Dadone (Gitarre), Philipe Garcia (Schlagzeug), Paolo Fresu (Trompete), Luciano Biondini (Akordeon), Bill Frisell (E-Gitarre), Greg Cohen (Kontrabass), Vittorio Piombo, Piero Salvatori, Sebastiano Severi (Cello), Piero Ponzo (Saxophon).

7 Zu dieser um 1990 entstandenen literarischen Strömung, für die abgesehen von dem anfangs stark im Vordergrund stehenden autobiographischen Aspekt die Merkmale des Translinguismus und der Transkulturalität konstitutiv sind, siehe u.a.: Gnisci 2003 u. 2006, Mengozzi 2013, Moll 2015.

8 De André wählt für sein Lied denselben Titel, aber in einer veränderten Schreibweise mit cedilla (einem Akzent der im Portugiesischen eigentlich nur beim ,c' vor dunklen Vokalen verwendet wird); unklar ist, ob dies aus stilistischen Gründen oder etwa aus urheberrechtlichen geschieht). 1997 entstand nach Vorlage desselben Romans der Dokumentarfilm Le strade di Princesa - ritratto di una trans molto speciale (unter der Regie von Stefano Consiglio).

9 Eine texttreue Vertonung dieses Gedichts wurde 2014 von dem „Canzoniere Grecanico“, einem Folkloreensemble aus dem süditalienischen Salento, realisiert und 2015 in dessen Album Quaranta aufgenommen.

10 Die Liedtexte werden aus der Buchausgabe von Da questa parte del mare (Testa 2016) zitiert.

11 Für weitere Verbindungen zwischen den literarischen Texten von Erri De Luca und den Liedertexten Testas siehe: Zoppi 2009, 7-45; für weitere typologische Bezüge zu Texten der italienischen Gegenwartsliteratur, in denen das Migrationsthema im Vordergrund steht, siehe: Lussignoli $2014 / 15$.

12 „In questi ultimi anni, però, alla sensazione di familiarità se n’è aggiunta un'altra. Il quarto stato è diventato per me un termine di paragone fra quella moltitudine in cammino e altre moltitudini contemporanee, anch'esse in cammino, ma senza quel passo e quello sguardo, perché a muoverle non è una volontà di giustizia, a muoverle è la disperazione di chi non ha più niente da perdere, la più forte delle energie“. Ivi, 6.

13 Dieser Begriff und die damit zusammenhängenden sozialen, kulturellen und auch musikalischen Hintergründe in den nordafrikanischen Ländern wurden von dem italienischen Schriftsteller und Aktivisten Gabriele del Grande in mehreren Vorträgen und Artikeln erläutert. Siehe dazu: www. fortresseurope.blogspot.it

14 „Non so se e quanto Jean-Claude abbia patito le sue origini meticce e straniere, forse soltanto il tempo dell'infanzia, o forse neppure quello, dal momento che lui era del ' 45 e suo padre era 
arrivato in Francia già nel '29. Non parlava l'italiano, e neanche, credo, lo spagnolo, forse dai suoi aveva ereditato non una lingua o una nazione di provenienza, dai suoi e da quella sua città aveva ereditato più semplicemente il Mediterraneo." (Testa 2016, 60)

15 Siehe zu diesem Thema die umfassende Studie von Eugenio Marino (Marino 2014). Dieses Buch verfügt auch über eine Website, die zahlreiche Angaben zu den zitierten Autorinnen und Autoren sowie Liedern enthält: www.andarsenesognando.it

16 Eine Stellungnahme zum politischen Gehalt seines Albums kann man in einem Interview von Lara Pischedda mit Testa nachlesen (Pischedda 2009); siehe ebenfalls: Marino 2014, 313-320.

17 Das von C.A. Bixio und B. Cherubini komponierte Lied ist u.a. in der Interpretation von Claudio Villa aus den Siebziger Jahren bekannt.

\section{Bibliographie}

De Luca, Erri: Solo andata. Righe che vanno troppo spesso a capo. Milano: Feltrinelli, 2005.

de Albuquerque, F. Farias / Jannelli, Maurizio: Princesa. Roma: Sensibili alle foglie, 1994 (deutsche Übersetzung: Princesa. Ein Stricherleben. Hamburg: Rotbuch Verlag, 1996).

Fracassa, Ugo: Patria e lettere. Per una critica della letteratura postcoloniale e migrante in Italia. Roma: Giulio Perrone editore, 2012.

Gnisci, Armando: Creolizzare l'Europa. Letteratura e migrazione. Roma: Meltemi, 2003.

Gnisci, Armando (Hg.): Nuovo planetario italiano. Troina: Città aperta, 2006.

Izzo, Jean-Claude: Marins perdus. Paris: Flammarion, 1997.

Lussignoli, Fabio: Da ogni parte del mare: storie di moderne migrazioni. Tesi di laurea in Letterature comparate. Roma: Facoltà di Scienze della Comunicazione, Università Telematica Internazionale Uninettuno, 2014/15.

Marino, Eugenio: Andarsene sognando. L'emigrazione nella canzone italiana. Isernia: Cosmo Iannone, 2014.

Menghozzi, Chiara: Narrazioni contese. Vent'anni di scritture italiane della migrazione. Roma: Carocci, 2013.

Moll, Nora: L'infinito sotto casa. Letteratura e transculturalità nella letteratura italiana contemporanea. Bologna: Pàtron, 2015.

Pischedda, Lara: „Intervista con Gianmaria Testa“. In: El Ghibli. Rivista on-line di letteratura della migrazione 4, 18 (2009), http://archivio.el-ghibli.org/index.php\%3Fid=1\&issue=04_19\&sectio $\mathrm{n}=6$ \&index_pos=2.html (Zugriff 20.06.2016).

Stella, Gianantonio: L'orda. Quando gli albanesi eravamo noi. Milano: Rizzoli, 2002.

Serra, Michele: „Intervista con Gianmaria Testa“. In: La Repubblica (11. Mai 2015), http://www. repubblica.it/cronaca/2015/05/11/news/gianmaria_testa_ho_un_tumore_ma_non_mi_fa_ paura_ora_penso_a_guarire_poi_torno_sul_palco_-114069870/ (Zugriff 20.06.2016).

Testa, Gianmaria: Da questa parte del mare. Torino: Einaudi 2016. 
Tirone, Paola / Giovannetti, Paolo: „Poesia e inganno nei cantautori anni '70“. In: Coveri, Lorenzo (Hg.): Parole in musica. Lingua e poesia nella canzone d'autore italiana. Novara: Interlinea edizioni, 1996, 115-142.

Zoppi, Isabella M.: Il giorno che passa e consuma. Storia, musica e parole di Gianmaria Testa. Civitella in Val di Chiana: Zona, 2007.

Zoppi, Isabella M.: „Da questa parte del mare: Gianmaria Testa ed Erri De Luca nel secolo delle migrazioni“. In: Altre Modernità 2 (10/2009), 37-45.

\section{Diskographie}

Testa, Gianmaria: Il valzer di un giorno. Harmonia mundi / Le chant du Monde B00005CCFR, 2002 (2000) (CD).

Testa, Gianmaria: Altre latitudini. Harmonia mundi / Le chant du Monde B01A9L5CY8, 2003 (CD).

Testa, Gianmaria: Da questa parte del mare. Harmonia mundi / Le chant du Monde B00I5YRO2, 2006 (CD). 